\title{
Impact of misclassification of Klatskin tumor on incidence of intra- and extrahepatic bile duct cancer: A population-based national cancer registry study
}

\author{
Mee Joo KANG', Jiwon LIM², Sung-Sik HAN', Hyeong Min PARK', Sun-Whe KIM', Young-Joo WON ${ }^{2,3}$, Sang-Jae PARK*1 \\ 'Department of Surgery, Center for Liver and Pancreatobiliary Cancer, National Cancer Center, Goyang, Korea \\ ${ }^{2}$ Division of Cancer Registration and Surveillance, National Cancer Center, Goyang, Korea \\ ${ }^{3}$ Department of Cancer Control \& Population Health, Graduate School of Cancer Science and Policy, National Cancer Center, Goyang, Korea
}

Introduction: Misclassification of Klatskin tumor as intrahepatic rather than extrahepatic bile duct cancer (eBDC) may overestimate the incidence of intrahepatic BDC (iBDC). However, the effect was insignificant in countries with lower incidence of BDC. Therefore, the impact of misclassified Klatskin tumor on iBDC and eBDC incidences was analyzed in a high endemic country of BDC.

Methods: The incidence and topography classification of morphology code "Klatskin tumor (8162/3)" from 1999 to 2017 were investigated based on data of the Korea Central Cancer Registry, which covers the entire 51.6 million Korean population. For misclassification analysis, all Klatskin tumor was reclassified as eBDC as proposed in ICD-O-3.2.

Results: Klatskin tumor accounted for $13.5 \%$ of all 81,414 BDC cases. In the registry, a mean of 59.7\% of Klatskin tumor was classified as iBDC, gradually decreasing from $95.5 \%$ to $16.9 \%$. Misclassification led to a $17.3 \%$ mean overestimation of iBDC cases and a mean underestimation of $15.0 \%$ eBDC cases. After reclassification, iBDC and eBDC constituted $46.4 \%$ and $53.6 \%$ of all BDC, respectively. Age standardized rate (ASR) of incidence per 100,000 population decreased in iBDC from 3.4 to 2.9 and increased in eBDC from 2.8 to 3.2 after reclassification. Average annual percent change of iBDC and eBDC incidences were 2.0\% and 1.2\%, respectively, but the ASR of iBDC significantly decreased since $2012(p<0.0001)$.

Conclusions: Misclassification of Klatskin tumor has remarkable impact on the incidence of iBDC and eBDC in a highly endemic country. The incidence of iBDC and eBDC increased over time but not linearly. 